\title{
Adult polycystic kidney disease and idiopathic dilated cardiomyopathy: a rare genetic association
}

\author{
Mariathasan D A L ${ }^{1}$, Kumanan $\mathbf{T}^{2}$ \\ Journal of the Ceylon College of Physicians, 2015, 46, 42-44
}

\begin{abstract}
Autosomal dominant polycystic kidney disease associated with dilated cardiomyopathy is a very rare association. Adult polycystic kidney disease is a systemic disorder from mutations in either $P K D-1$ or $P K D-2$ gene, that encode the proteins polycystin-1 (PC1) and polycystin-2 (PC2) respectively. Polycystin-2 is an intracellular calcium channel expressed in both renal epithelial cells and cardiac myocytes. It regulates intracellular calcium cycling. Mutation causes impaired intracellular calcium cycling and contributes to heart failure. Those who have $P K D-2$ mutation can have polycystic kidneys associated with idiopathic dilated cardiomyopathy.
\end{abstract}

Key words: adult polycystic kidney disease; idiopathic dilated cardiomyopathy; $P K D-2$ gene

\section{Case report}

A 40-year-old male was presented with gradual worsening of abdominal distension, bilateral leg swelling and difficulty in breathing of 2 weeks duration. Except the fact that he had a polio affected left leg, he had led a healthy life. He had no siblings and there was no family history of heart disease or hypertension. He was neither an ethanol abuser nor a smoker.

On examination he was tachypnoeic; had bilateral pitting ankle oedema; bilateral basal fine crackles and elevated jugular venous pressure. His blood pressure was $130 / 90 \mathrm{mmHg}$. He had flank fullness with ballotable mass in the left lumbar region. The oxygen saturation was $93 \%$ on room air. His complete blood count was normal. Chest radiograph revealed a massive car-

\footnotetext{
${ }^{1}$ Registrar in Medicine,

${ }^{2}$ Consultant Physician, University Medical Unit, Teaching Hospital, Jaffna, Sri Lanka.

Corresponding author: DALM, E-mail: dharmikaanoj@gmail.com
}

diomegaly with features of pulmonary oedema. Urine analysis showed albumin of 1+ with 1-2 red cells/HPF. With intravenous furosemide, his respiratory distress improved and oxygen saturation was maintained at $96 \%$ on room air. His renal and liver functions were normal. His ECG showed inverted T waves in inferior and lateral leads with no evidence of left ventricular hypertrophy (Figure 3). His cardiac biomarkers were normal.

Ultrasound scan of the abdomen was performed which revealed bilateral enlarged kidneys with multiple cysts in cortex and medulla suggestive of polycystic kidney disease. No liver cysts were noted. 2D Echocardiogram showed a globally hypokinetic heart with severe left ventricular dysfunction (Ejection fraction $30 \%$ ) suggestive of dilated cardiomyopathy. Workups to exclude the aetiology for cardiomyopathy including serum ferritin, thyroid function were unremarkable. With treatment, he showed marked symptomatic improvement.

\section{Discussion}

This case report illustrates an association between autosomal dominant polycystic kidney disease and dilated cardiomyopathy.

Autosomal dominant polycystic kidney disease (ADPKD) occurs in 1:400 - 1:1000 individuals worldwide. It is a systemic disorder resulting from mutations in either $P K D-1$ or $P K D-2$ genes, which encode the proteins polycystin-1 (PC1) and polycystin-2 (PC2) respectively. Polycystin-2 is important for intra cellular calcium cycling especially in renal epithelial cells and cardiac myocytes. Mutation in PKD-1 gene and $P K D-2$ gene occurs in chromosome 16 and 4 respectively.

Affected individuals are asymptomatic until the fourth or fifth decade. Renal function declines progressively over 10-20 years and end stage renal disease ensues in about 60\%. Extra renal manifestations of this systemic disorder are intracranial aneurysms, cardiac valvular abnormalities such as mitral valve prolapse and aortic regurgitation, hepatic cysts, colonic diverticulae and abdominal wall and inguinal herniae. Cardiomyopathy is usually observed 

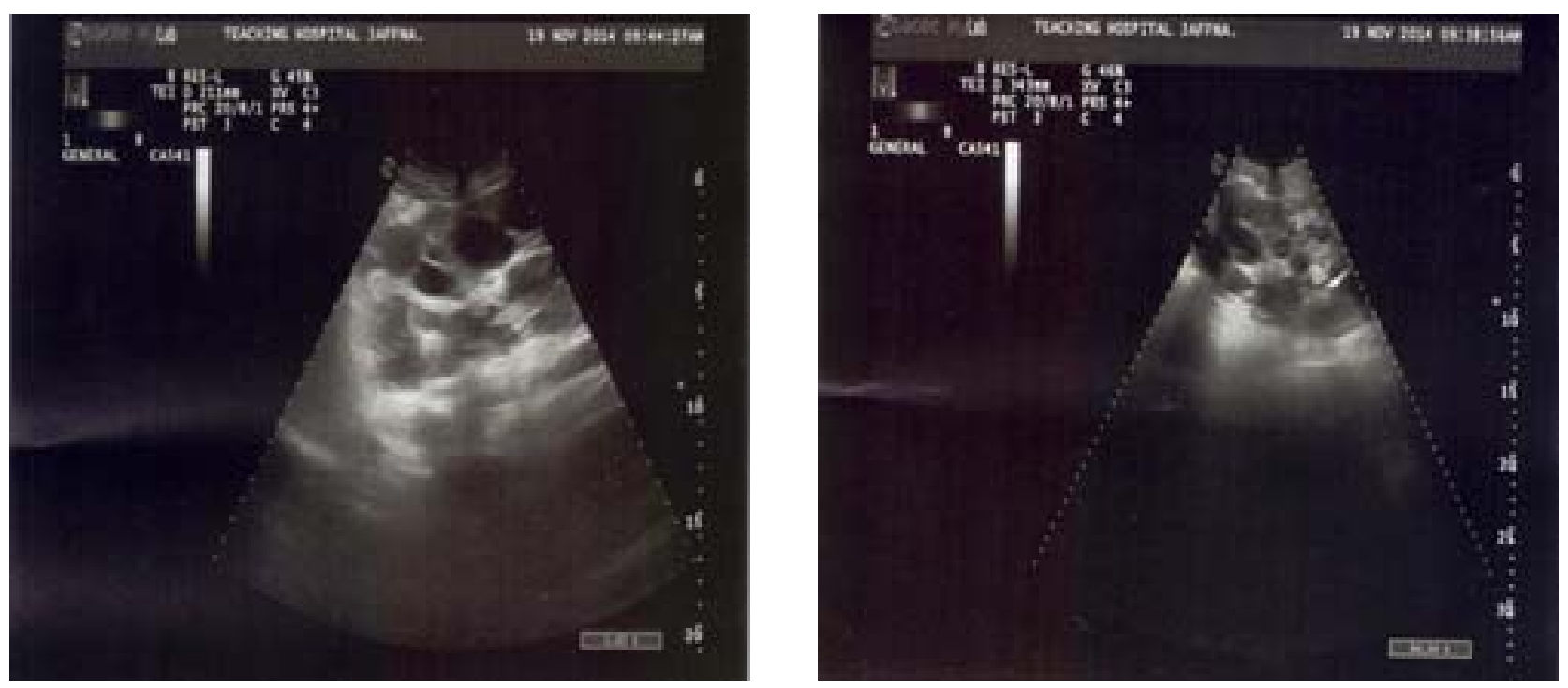

Figure 1. (a) and (b): Ultrasound scan of right and left kidneys shows multiple cysts
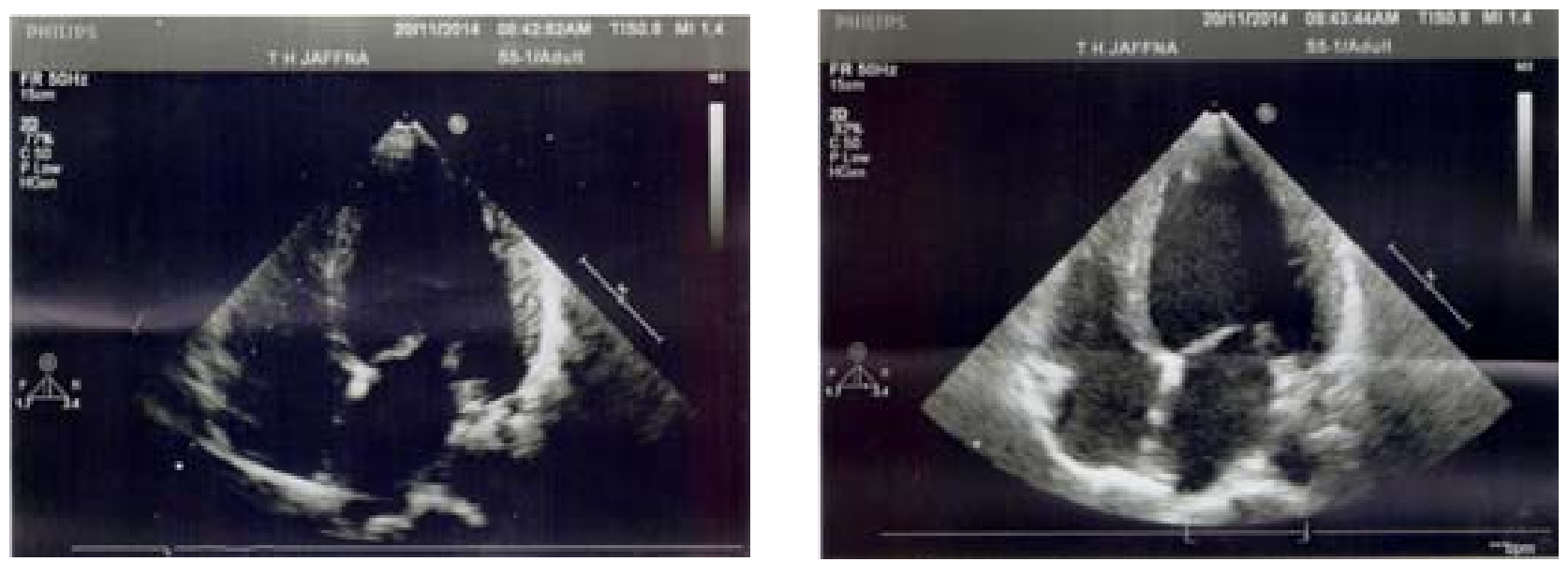

Figure 2. (a) and (b): 2D echocardiogram demonstrates dilatation of all four cardiac chambers
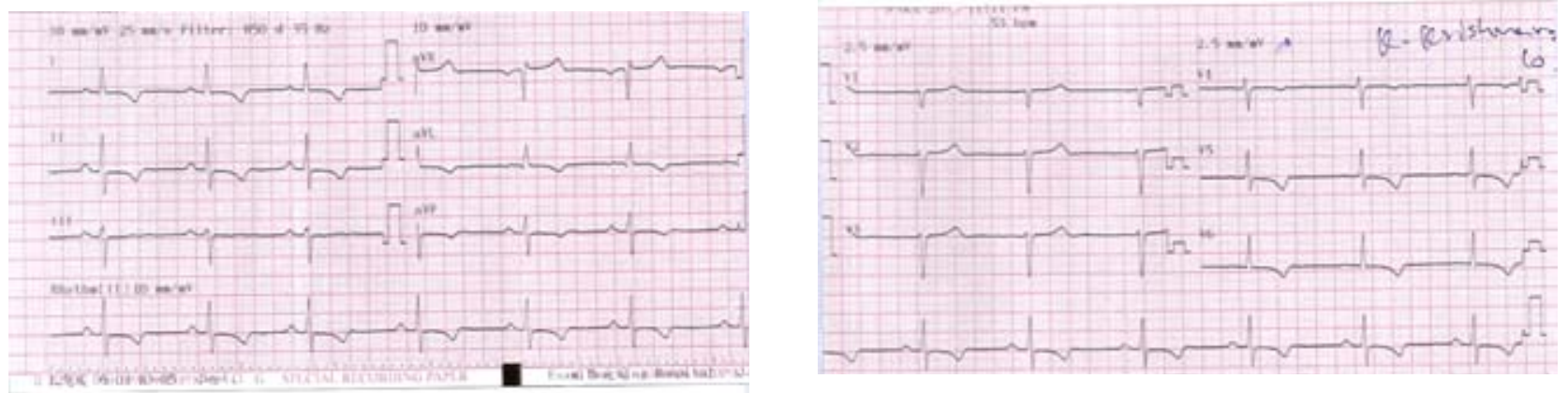

Figure 3.

in patients with end stage renal disease due to uraemia. As ischaemic heart disease is a common complication and contributes to mortality in renal failure, ischaemic cardiomyopathy should also be considered in the differential diagnosis.
Long standing hypertension also leads to dilated cardiomyopathy. The absence of left ventricular hypertrophy on ECG or 2D echocardiogram and an unremarkable funduscopy rules out its possibility. In the absence of above mentioned complications and 
the negative preliminary work up to exclude the possible cause of secondary cardiomyopathy, we can reasonably conclude that the cardiomyopathy in this patient is idiopathic. The earliest case reported from Korea in the year $2001^{1}$ and PKD-2 mutant study ${ }^{2}$ in 2013 describe, idiopathic dilated cardiomyopathy associated with autosomal dominant polycystic kidney disease in those who have mutation in $P K D-2$ gene.

In conclusion, this gentleman was diagnosed to have adult polycystic kidney disease and the rare association of dilated cardiomyopathy and urther genetic studies are warranted to confirm the presence of $P K D-2$ gene mutation.

\section{References}

1. Choe SC, Jeon ES, Hwang SD. A Case of Dilated Car- diomyopathy Associated with Autosomal Dominant Polycystic Kidney Disease. Korean Soc Echocardiogr 2001; 9: 152-6.

2. Paavola J, Schliffke S, Rossetti S, Kuo IY, Yuan S, Sun Z, Harris PC, Torres VE, Ehrlich BE. Polycystin-2 mutations lead to impaired calcium cycling in the heart and predispose to dilated cardiomyopathy. Mol Cell Cardiol 2013; 58: 199-208.

3. Patricia D Wilson. Polycystic Kidney Disease. The New England Journal of Medicine 2004; 350: 151-64.

4. Riccardo Lubrano, Paolo Versacci, Giuliana Guido, Elena Bellelli, Giammarco Andreoli Marco Elli. Might there be an association between polycystic kidney disease and non compaction of the ventricular myocardium. Oxford Journals 2009; 24: 3884-6. 